\title{
A novel accessory muscle in the flexor compartment of anterior forearm inserting into the tenosynovium of the flexor pollicis longus
}

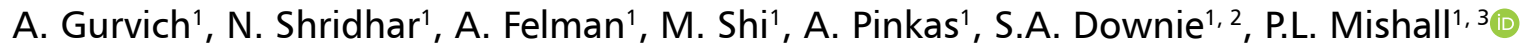 \\ ${ }^{1}$ Department of Anatomy and Structural Biology, Albert Einstein College of Medicine, Bronx, NY, United States \\ ${ }^{2}$ Department of Physical Medicine and Rehabilitation, Albert Einstein College of Medicine, Bronx, NY, United States \\ ${ }^{3}$ Department of Ophthalmology and Visual Sciences, Albert Einstein College of Medicine, Bronx, NY, United States
}

[Received: 5 March 2021; Accepted: 18 March 2021; Early publication date: 9 April 2021]

\begin{abstract}
A common variant of accessory muscles in the anterior forearm is the Gantzer's muscle (GM). GM arises as a muscle belly from flexor digitorum superficialis (FDS) or ulnar coronoid process to merge distally with the flexor pollicis longus (FPL) muscle. In the present case report, we describe a novel accessory muscle in the flexor compartment of the forearm. The proximal attachment was tendinous and came from three sources: FDS muscle, ulnar coronoid process, and the medial aspect of the proximal radius. The distal tendon of the novel accessory muscle ran parallel to FPL, passed through the carpal tunnel, and entered the palmar aspect of the hand. In the hand, the tendon thinned out and blended with the tenosynovium of the FPL, contributing to the sheath around the FPL tendon. This accessory muscle of the FPL is comparable to the frequently documented GM; however, the present case exhibited fundamental nuances that distinguish it from the previously described iterations of the GM in the following ways: 1) The novel accessory muscle is tendinous from its proximal origin and throughout the upper one-third of the forearm, and one component of its origin arose from the medial aspect of the radius. GMs with an origin on the radius have not been previously reported. 2) In the middle one-third, the tendinous proximal attachment transitioned to a muscle belly that passed through the carpal tunnel and entered the hand. 3) In the hand, the novel tendon widened, thinned, and merged with the tenosynovium of the FPL. Accessory muscles are a common finding in the anterior forearm during cadaveric dissection. In patients, they can be the cause of neuropathies due to compression of the anterior interosseous nerve. Awareness of variations is also important for clinicians who examine the forearm and hand, as well as hand surgeons. (Folia Morphol 2022; 81, 2: 515-519)
\end{abstract}

Key words: Gantzer, forearm, flexor pollicis longus, carpel tunnel

\section{INTRODUCTION}

Anatomical variations of the flexor muscles of the anterior forearm are commonly found in cadaveric dissections. The Gantzer muscle (GM), accessory head of the flexor pollicis longus (FPL), is a well-documented and highly prevalent anterior compartment variation. One meta-analysis reported a prevalence rate of $44.2 \%$ from 24 cadaveric studies [11]. Typically, the

Address for correspondence: P.L. Mishall, Ass. Prof., Departments of Anatomy and Structural Biology and Ophthalmology and Visual Sciences, Albert Einstein College of Medicine, 1300 Morris Park Ave, F620SD, Bronx, NY 10461, United States, tel: +1 (718) 430-3423,

fax: +1 (718) 430-8996, e-mail: priti.mishall@einsteinmed.org

This article is available in open access under Creative Common Attribution-Non-Commercial-No Derivatives 4.0 International (CC BY-NC-ND 4.0) license, allowing to download articles and share them with others as long as they credit the authors and the publisher, but without permission to change them in any way or use them commercially. 
GM originates in the proximal forearm as fibres from the deep aspect of the flexor digitorum superficialis (FDS) alone, or from the FDS together with the medial epicondyle of the humerus and/or with the ulnar coronoid process [10]. Distally, GMs blend in with the proximal tendon of the FPL at the midpoint of the radius. At that insertion, they exert a flexion force on the FPL. Also reported are accessory flexor digitorum profundus (FDP) muscles which have the same origin as the GM but insert into the FDP [15]. Some variants insert into both the FPL and the FDP [15].

Accessory muscles in the anterior forearm become clinically relevant when they compress local neurovasculature. An accessory head of the FPL has been the aetiology of compressive neuropathies of either the median nerve (MN) or the anterior interosseous nerve $[13,15]$. Here, we present a muscle that would raise similar concerns in the clinical setting.

\section{CASE REPORT}

An 84-year-old female donor was dissected by undergraduate medical students at the Albert Einstein College of Medicine, Bronx, NY, as part of the first-year Clinical and Developmental Anatomy course. They identified a novel accessory muscle in the anterior flexor compartment of the right forearm. It originated via tendinous bands from the deep side of the FDS, the ulnar coronoid process, and the medial aspect of the proximal radius. These two tendinous bands united at the upper one-third of the forearm to form a single tendon (Fig. 1). These proximal tendons were positioned anterior to the anterior interosseous nerve (AIN). In the distal half of the forearm, the single tendon transitioned into a muscle belly located deep to the FDS and between the FDS and the MN. The muscle again became tendinous at the level of the radial styloid process. This distal tendon was situated between the FDS and the MN, and ran parallel to the FPL (Figs. 2, 3). It passed through the carpal tunnel and entered the hand, where it widened out and blended with the tenosynovium of the FPL (Figs. 4, 5).

The dissection of the left anterior forearm revealed an accessory head of FPL that originated from the deep side of the FDS and inserted into the FPL tendon as has been described previously [2].

\section{DISCUSSION}

The accessory muscle reported here is atypical of GMs and other accessory muscles of the forearm. This novel muscle's proximal origins - contributions from

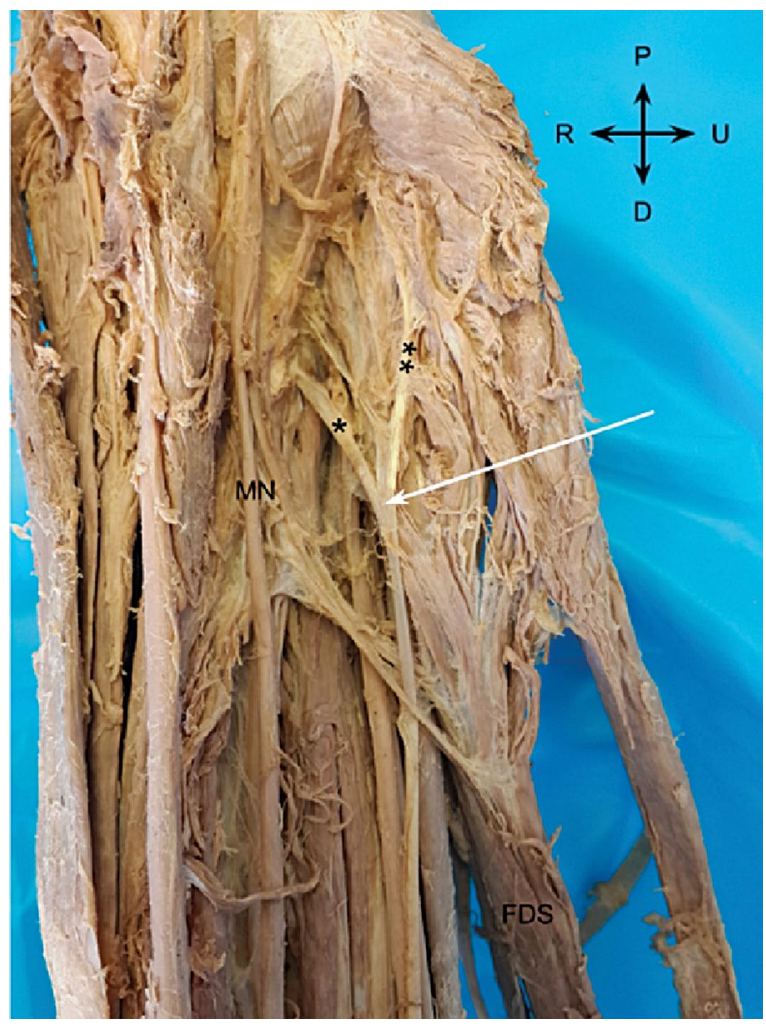

Figure 1. Dissection of the anterior proximal forearm. The novel muscle (white arrow) seen with its origins in the medial aspect of the radius $\left({ }^{*}\right)$ and the ulnar coronoid process $\left({ }^{* *}\right)$, with contributory fibres from the flexor digitorum superficialis (FDS), converging in the form of a " $Y$ ". The position of the muscle is shown relative to the median nerve (MN).

the undersurface of the FDS, the ulnar coronoid, and the proximal medial radius - have not been previously reported. Pai et al. [10] surveyed 126 upper limbs and found that 76 had a GM, either as an accessory head of FDP (FDPah) or of FPL (FPLah). In all cases, the origin was from the deep surface of the FDS alone, or in combination with fibres originating on the medial epicondyle and/or the coronoid process. In no cases did the GM originate on the coronoid process or medial epicondyle without the FDS contribution. Pai et al. [10] also writes they found no muscle arising from other structures such as the pronator teres or the brachialis. The novel muscle is most similar to one case Pai et al. [10] found, involving a single muscle belly of a GM that originated from the FDS, and then divided into an FPLah and an FDPah to insert into the FPL and FDP, respectively.

Caetano et al. [2] conducted a survey of 80 upper limbs and found GM in 54 (68\%). In 42 of these cases the GM originated from the undersurface of the FDS, in 8 cases it originated from the coronoid process, 


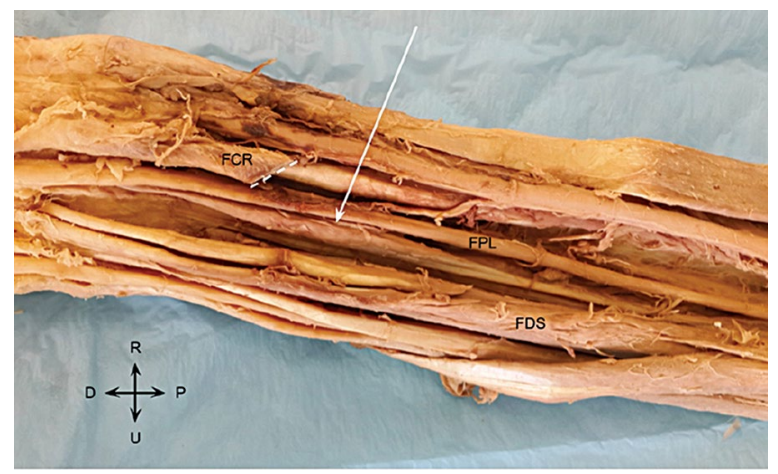

Figure 2. Dissection of the distal forearm. The flexor carpi radialis (FCR) was cut (at dashed line) from its distal attachment and reflected to better visualise the flexor pollicis longus (FPL). The novel muscle is denoted by the white arrow, medial to the FPL. The flexor digitorum superficialis (FDS) remains in situ.

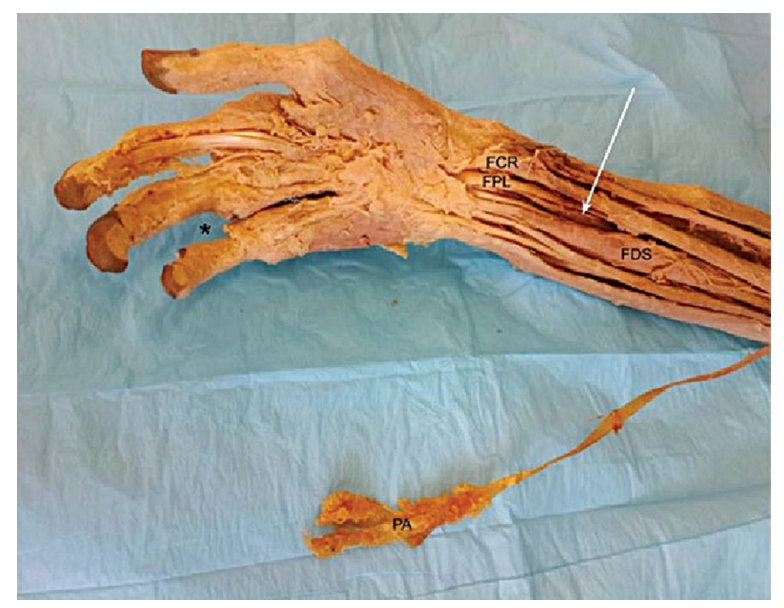

Figure 3. Dissection of anterior compartment of right distal forearm and hand. The palmaris longus, with the palmar aponeurosis (PA), was reflected. The flexor digitorum superficialis (FDS), flexor pollicis longus (FPL), and flexor carpi radialis (FCR) are shown in situ. White arrow denotes the novel muscle, medial to $\mathrm{FPL} ;{ }^{*}$ (asterisk) - the fourth digit was surgically amputated in life.

and in 7 cases from the medial epicondyle ( 3 of the cases were a GM with 2 origins, both the FDS and the coronoid process). In all of the cases Caetano et al. [2] examined, the GM always lay posterior to both the median and anterior interosseous nerve.

Regarding the distal insertion of the GM, Pai et al. [10] conducted a cadaveric survey of 126 upper limbs and found that GMs inserted into the FPL at a prevalence of $46.03 \%$, and into the FDP at that of $14.28 \%$. Among those GMs positioned as FPLah, $80 \%$ inserted into the proximal third of the FPL, and the rest mostly inserted into the middle third, except for 2 cases in which the FPLah inserted into the distal third of the FPL. No cases were found in which FPLah inserted into the tendon of the FPL distal to the carpal tunnel. Similarly, Zdilla et al. [15] reported a belly of a GM which became tendinous at $52 \mathrm{~mm}$ from its most distal origin in the FDS; this tendon travelled $6 \mathrm{~mm}$ before joining the proximal one third of the FPL.

In the study conducted by Caetano et al. [2], the GM inserted into the FPL in 36 of the 54 limbs. It inserted into the FDP in 21 of the upper limbs, totalling 57 insertions in 54 limbs (in 3 cases of GM that inserted into the FDP, the GM had 2 origins, and therefore was counted twice).

There have been various descriptions in the literature of the location of the GM relative to the MN and AIN. Mangini [8] found that the FPLah lies posterior to the $\mathrm{MN}$ and anterior to the AIN in $100 \%$ of his cases, and this finding was confirmed by Pai et al. [10]. However, Al-Qattan [1] found in 13 cadavers that the FPLah lies posterior to the AIN, as was described by Dellon and Mackinnon [4] in all 14 cadavers with a GM present. In this case report, the novel muscle was found to lie posterior to the $\mathrm{MN}$, and anterior and parallel to the AIN, in line with the findings of Mangini [8] and Pai et al. [10].

The accessory head of FPL has been implicated in the compression of the AIN $[4,14]$. This has been termed Kiloh-Nevin syndrome or AIN syndrome. Spinner [14] describes this compression as causing AIN paralysis, thus affecting the FPL, the lateral two fascicles of FDP, and pronator quadratus muscles. Clinically, this syndrome presents with increased extension of the distal interphalangeal joint and increased flexion of the proximal interphalangeal joint on the index finger during the pinch motion. This has been dubbed "Spinner's sign" [3], and the accessory head of the FPL has been implicated in this syndrome. However, per the literature review of Orellana-Donoso et al. [9], a compressive neuropathy of the AIN by an accessory head of the FPL mainly presents clinically as carpal tunnel syndrome. Alternatively, the presence of an anomalous muscle or tendon in the forearm has been implicated in symptomatic supernumerary muscle belly syndrome [12]. This presents as pain in the distal forearm due to shearing between adjacent muscle fibres as a result of the extra muscle or tendon in the forearm [12].

The musculature of the human anterior forearm has its embryological origins in somatic mesoderm which invades the limb anlage at 4 weeks of gestation. The flexor mass subsequently divides into 


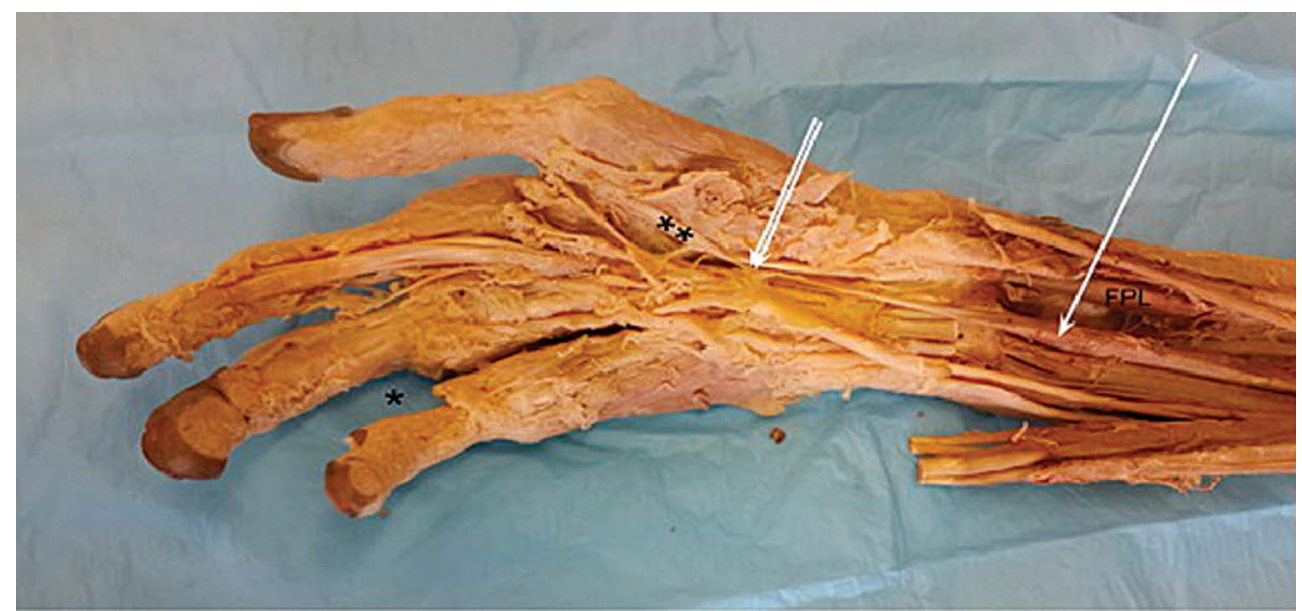

Figure 4. Dissection of palm of hand. The novel muscle (single arrow) separated from the flexor pollicis longus (FPL). The transverse carpal ligament was dissected to visualise the distal continuity of these two muscles. The tendon of the novel muscle distally feeds into the tenosynovium of the FPL (double arrows) immediately distal to the carpal tunnel, forming a sheath (double asterisk); * (asterisk) — the fourth digit was surgically amputated in life.

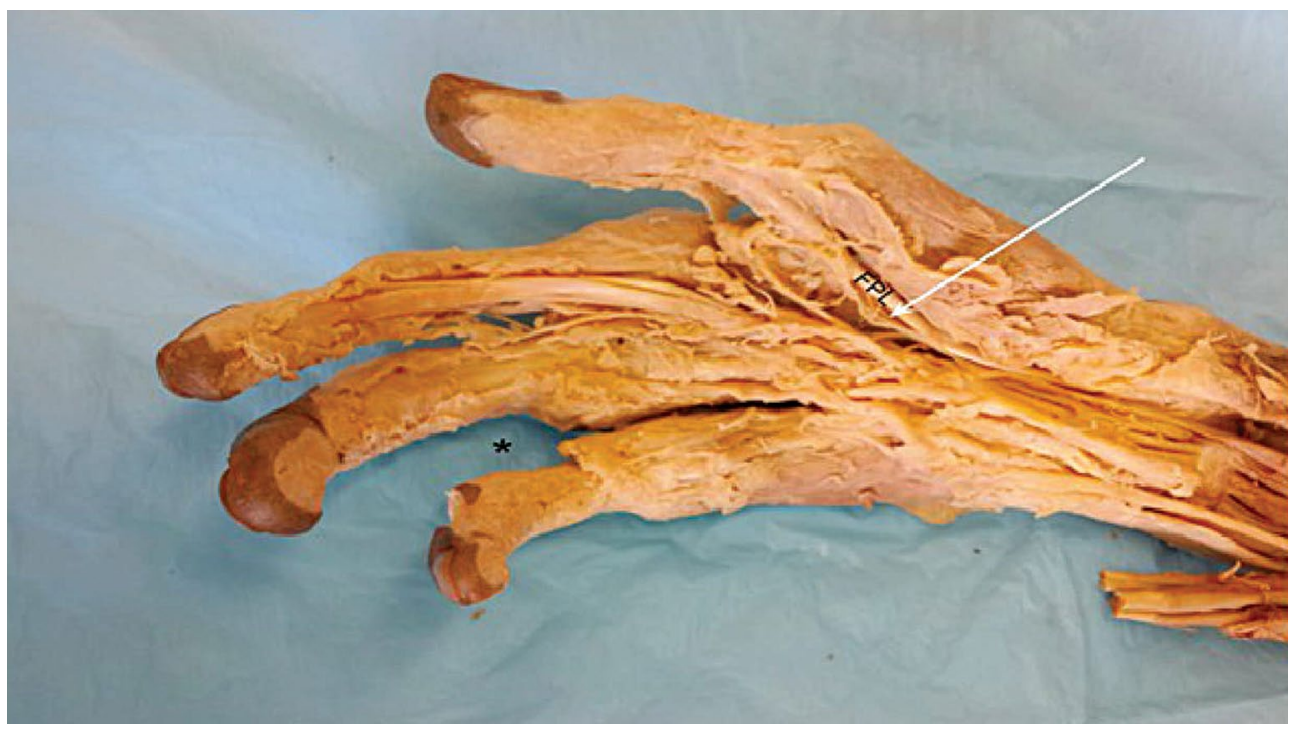

Figure 5. Deep dissection of palm of hand. Sheath formed by tendon of novel muscle (arrow) further dissected from the tendon of the flexor pollicis longus (FPL); * (asterisk) — the fourth digit was surgically amputated in life.

two layers that condense: one more superficial, and another deeper. The former splits into flexor carpi radialis, pronator teres, and the palmaris longus. The deep muscle mass originates on the medial epicondyle and inserts into the developing digits. It initially gives rise to the flexor carpi ulnaris and then cleaves into two layers: the FDS and the FDP. The FPL splits from the FDP [7]. Accessory muscles that connect the FDS, FDP, and FPL are thought to be the result of incomplete cleavage of the deeper embryological mass in the anterior forearm [6]. According to Eid and Otsuki [5], an accessory head of the flexor compartment, in other mammals, is a belly connecting the part of FDS originating in the medial epicondyle to the deep flexor muscles. These observations of FPL splitting from FDP are consistent with our findings of the novel muscle originating from deep side of FDS, although they do not address the unique origin of the novel muscle fibres from medial aspect of radius.

The accessory muscle in the right forearm identified by the authors differs from previously described GMs in three ways: 
- this accessory muscle had one origin from the medial aspect of the radius. GMs with an origin on the radius have not been previously reported;

- this accessory muscle presented as a muscle belly in the middle of the forearm. The tendon of the belly passed through the carpal tunnel and travelled further into the hand. Even the most distally inserted GMs found by Pai et al. [10] inserted into the FPL proximal to the carpal tunnel;

- in the hand, the distal tendon of the accessory muscle formed a synovial sheath around the tendon of the FPL medial to the thenar eminence. This is a previously unreported finding.

\section{CONCLUSIONS}

To our knowledge, this is the first report of an accessory muscle in the anterior forearm with a proximal origin that included the radius. The belly of this muscle transitioned into a tendon at the radial styloid process, entered the hand through the carpal tunnel, and fused with the tenosynovial sheath of the FPL. GM usually inserts into the FPL tendon in the middle third of the forearm. More generally, the presence of accessory muscles such as the one described here has clinical implications for the diagnosis and treatment of compression injuries of the median and anterior interosseous nerves.

\section{Acknowledgements}

The authors acknowledge and gratefully thank the individual whose body and tissues were used in this study for the advancement of physician education and patient care.

\section{Conflict of interest: None declared}

\section{REFERENCES}

1. Al-Qattan MM. Gantzer's muscle. An anatomical study of the accessory head of the flexor pollicis longus muscle. J Hand Sur (Br). 1996; 21: 269-270, doi: 10.1016/s02667681(96)80114-8, indexed in Pubmed: 8732417.

2. Caetano EB, Sabongi JJ, Vieira LÂ, et al. Gantzer muscle. An anatomical study. Acta Ortop Bras. 2015; 23(2): 72-75, doi: 10.1590/1413-78522015230200955, indexed in Pubmed: 27069404.
3. Degreef I, De Smet L. Anterior interosseous nerve paralysis due to Gantzer's muscle. Acta Orthop Belg. 2004; 70(5): 482-484, indexed in Pubmed: 15587039.

4. Dellon AL, Mackinnon SE. Musculoaponeurotic variations along the course of the median nerve in the proximal forearm. J Hand Surg (Br) . 1987; 12: 359-363, doi: 10.1016/0266-7681(87)90189-6, indexed in Pubmed: 3437205.

5. Eid N, Otsuki Y. A case of double Gantzer's muscle and its possible role in nerve entrapment. Clin Anat. 2009; 22(8): 881-882, doi: 10.1002/ca.20862, indexed in Pubmed: 19806672.

6. Jones M, Abrahams PH, Sañudo JR, et al. Incidence and morphology of accessory heads of flexor pollicis longus and flexor digitorum profundus (Gantzer's muscles). J Anat. 1997; 191 ( Pt 3): 451-455, doi: 10.1046/j.14697580.1997.19130451.x, indexed in Pubmed: 9419002.

7. Lewis WH. The development of the muscular system. Manual of Embryology. 1910; 2: 455-522.

8. Mangini U. Flexor pollicis longus muscle. Its morphology and clinical significance. J Bone Joint Surg Am. 1960; 42(A): 467-470, indexed in Pubmed: 13853793.

9. Orellana-Donoso M, Valenzuela-Fuenzalida J, GoldSemmler $\mathrm{M}$, et al. Neural entrapments associated with musculoskeletal anatomical variations of the upper limb: Literature review. Transl Rev Anat. 2021; 22: 100094, doi: 10.1016/j.tria.2020.100094.

10. Pai MM, Nayak SR, Krishnamurthy A, et al. The accessory heads of flexor pollicis longus and flexor digitorum profundus: Incidence and morphology. Clin Anat. 2008; 21(3): 252-258, doi: 10.1002/ca.20612, indexed in Pubmed: 18351652 .

11. Roy J, Henry BM, Pękala PA, et al. The prevalence and anatomical characteristics of the accessory head of the flexor pollicis longus muscle: a meta-analysis. Peer J. 2015; 3: e1255, doi: 10.7717/peerj.1255, indexed in Pubmed: 26557419.

12. Ryu JY, Watson HK. SSMB syndrome. Symptomatic supernumerary muscle belly syndrome. Clin Orthop Relat Res. 1987(216): 195-202, indexed in Pubmed: 3815948.

13. Sookur PA, Naraghi AM, Bleakney RR, et al. Accessory muscles: anatomy, symptoms, and radiologic evaluation. Radiographics. 2008; 28(2): 481-499, doi: 10.1148/ rg.282075064, indexed in Pubmed: 18349452.

14. Spinner M. The anterior interosseous-nerve syndrome, with special attention to its variations. J Bone Joint Surg Am. 1970; 52(1): 84-94, doi: 10.1007/s12565-018-0466-6, indexed in Pubmed: 5411776.

15. Zdilla MJ, Pacurari P, Celuck TJ, et al. A Gantzer muscle arising from the brachialis and flexor digitorum superficialis: embryological considerations and implications for median nerve entrapment. Anat Sci Int. 2019; 94(1): 150-153, doi: 10.1007/s12565-018-0466-6, indexed in Pubmed: 30382571. 